\title{
Front end electronics for European XFEL sensor: the AGIPD project
}

\author{
A. Marras ${ }^{\text {a,* }}$, U. Trunk ${ }^{\text {a }}$, A. Klyuev ${ }^{\text {a }}$, J. Becker ${ }^{\text {a }}$, \\ H. Graafsma ${ }^{\text {a,c }}$, X. Shi ${ }^{\text {b }}$, D. Griffenberg ${ }^{b}$, B. Schmitt ${ }^{b}$ \\ a Deutsches Elektronen-Synchrotron, Hamburg, Germany \\ ${ }^{\mathrm{b}}$ Paul Schrerrer Institute, Villigen, Switzerland \\ ${ }^{\mathrm{c}}$ Mid Sweden university, Sundvall, Sweden
}

\begin{abstract}
8 Abstract
\footnotetext{
* Corresponding author.

Email address: alessandro.marras@desy.it (A. Marras).
} 
pulses (up to 2700 pulses in $0.6 \mathrm{~ms}$ ) separated by a period of $99.4 \mathrm{~ms}$. Each pulse will be around about $100 \mathrm{fs}$ long. This means that the front end has to cope with a high dynamic range, while having a noise low enough to discriminate single photons. Photon counting cannot be used (because of the high flux per pixel), and the sensor is required to provide a way to store the information from several (ideally, all) pulses on-board, to be read out in the interval between trains. At the same time, it also has to be substantially radiation-hard. The AGIPD (Adaptive Gain Integrating Pixel Detector) system [2] is being developed as a way to cope with such challenges. The development is shared between DESY, PSI, the universities of Hamburg and Bonn.

\section{2 sensor overview}

The system will consists in a 1Mpixel hybrid pixel detector, featuring a pitch of $200 \mu \mathrm{m}$. The front face of the silicon sensor will be composed of 16 modules each having a size of about $100 \mathrm{mmx} 26 \mathrm{~mm}$, without any gaps or other dead area (although there is a $3 \mathrm{~mm}$ gap between the modules). Each module is composed of $2 \times 8$ arrays of $64 \times 64$ pixels, each array being bump bonded to its own ASIC. Each ASIC will thus also include 64x64 pixels with a 200um pitch, and inside each pixel circuits for signal processing and storage are imbedded. The charge collected by the sensor is integrated by an adaptive preamplifier, and then fed to a Correlated Double Sampling stage. The information obtained is then stored in an analog memory bank inside the pixel, along with the gain value used in the preamplifier. All this must happen in 220ns, so that the system is ready to record a frame coming from the next X-ray pulse. When the pulse train has expired, those data can be red sequentially through an output chargesensitive buffer, using a dual column parallel readout (Fig. 1). Readout ASICs are to be manufactured in IBM $130 \mathrm{~nm}$ CMOS technology, and are foreseen to dissipate about $2 \mathrm{~W} /$ chip. Several test prototypes have been produced on a reduced scale (16x16 pixels), by means of MPW runs, both to test the technology characteristics and to evaluate the best architectural solutions to be employed. Radiation-hard design techniques are employed, with the use of Enclosed Layout Transistors and guard rings around the critical devices. Most of the issues have been settled, and we foresee to have the ASIC for the 1Mpixel system to be ready for submission end-2012.

\section{3 answering the XFEL challenges}

The large dynamic range and single photon sensitivity constraints coming from the XFEL source were solved with a adaptable charge amplifier [3] integrated 
inside each pixel, having a gain ranging over 2 orders of magnitude, dynamically adjustable in real-time to the number of absorbed photons. Basically, it is a charge-sensitive amplifier, with a battery of different capacitors which can be connected to it. The system starts integrating charge at its maximum gain, so that single photon sensitivity is guaranteed in low flux condition. If the amplifier output exceeds a certain level, however, a discriminator is triggered, causing an additional feedback capacitor to be connected in parallel with the original one. Thus the gain is lowered, allowing charge integration on a higher dynamic range without loosing any of the already integrated charge. Three capacitors of $60 \mathrm{fF} / 3 \mathrm{pF} / 10 \mathrm{pF}$ permit a gain reduction up to two orders of magnitude, thus allowing for a dynamic range of $1-1000012.4 \mathrm{keV}$ photons. The output of the CDS stage is written into an analogue memory array embedded into the pixel, along with its gain setting, encoded as an analogue 3-levels signal. Thus the basic memory cell consists actually of a couple of storage cells, one for the signal and one for the gain settings info [4]. In the most recent (AGIPD04) test prototype we were able to embed 32x11 of such structures in a pixel, thus achieving a 352 memory depth (Fig. 2). This is certainly less than the ideal case, since up to 2700 images are produced during a XFEL pulse train, but it comes as a compromise between keeping pixel dimensions reduced, leakage minimization and radiation hard design issues. As a partial solution of the limited memory depth problem, the memory array and its control logic have been designed such that the memory space is addressable RAM-like from the (external) interface electronics. This allows for the so-called veto scheme, consisting in the possibility of overwriting the memory cells containing meaningless data (as not every X-ray pulse will overlap with the sample in time and space, and some time will be needed to remove debris after single-shot experiments involving sample destruction), that can thus be re-used overwriting meaningul information in them. Other than the pixel matrix, the ASIC has a control digital logic to address the pixels and their memory cells, synthetized using the technology provided ARM cmrf8sf RVT Standard Cell Library. The control logic is based on a Command Serial interface using 3 Low Voltage Differential Signals generated by the interface electronics as a clock, a signal to communicate the pulse arrival and a 16-bit command line to be used at a frequency between 80 and $160 \mathrm{MHz}$. Several pixel flavours having different circuital variations were developed, manufactured by means of MPW, and tested, allowing an optimal configuration to be selected. Encouraging results comes from X-rays detection using a 16x16 prototype chips, even if used in sub-optimal condition (at room temperature and with a non-optimized sensor): total noise is estimated as 300-320e, less that $1 / 10$ of a photon having a $12.4 \mathrm{keV}$ energy (Fig. 3). Positive responses were obtained from the system using Mo target (Fig. 4), as well as when illuminated with a $7 \mathrm{keV}$ beam (Petra III P10 beam facility at Desy) and with $20-35 \mathrm{keV} \mathrm{X}$ ray tubes. An irradiation campaign has been performed, using the Doris F4 irradiation facility at Deutsches Elektronen-Synchrotron, and exposing prototypes (AGIPD03,AGIPD04) to a dose of 1, 5, 10 and 100MGy. 
The ASIC was found to be rad-tolerant up to at least 10 MGy: performance of irradiated samples up to that dose is comparable to that of non-irradiated samples, showing only a limited noise increase which saturates above 1MGy (Fig. 5). When increasing dose from 10 to $100 \mathrm{MGy}$, the ASIC stops working, most probably because of charge accumulation in the oxide layer which causes substantial shift of the threshold voltages of the devices. The charge gradually wears off (a process which could be accelerated by thermal annealing), after which even 100MGy-irradiated systems recover functionality, albeit with a reduced analog performance which is still under investigation.

\section{Conclusion}

A 1 Mpixel AGIPD sensor is being developed jointly by DESY, PSI, the universities of Hamburg and Bonn, to be used in the European X-ray FreeElectron Laser, several reduced-dimension prototypes have been produced for test purposes using MPWs The main characteristics of the system are: the presence of an adaptive gain stage to guarantee a good dynamic range; an in-pixel Memory embedded memory able to store 350 frames; on-chip control logic. The system has a noise evaluated in 300-320 electrons, thus allowing for single photon resolution in XFEL experiments (involving $12.4 \mathrm{keV}$ photons). Radiation and leakage issues are under investigation but the ASIC is radtolerant at least up to 10MGy The ASIC for the 1Mpixel system is foreseen to be ready next year

\section{References}

[1] www.xfel.eu

[2] hasylab.desy.de/instumentation/detectors/projects/AGIPD

[3] X. Shi et al., "Challenges in chip design for the AGIPD detector", Nuclear Instruments and Methods-A, Volume 624, Issue 2, 11 December 2010, Pages 387-391

[4] U. Trunk et al., "AGIPD - The adaptive gain integrating pixel detector for the European XFEL development and status" Nuclear Science Symposium 2011, Pages: 1950 - 1954 


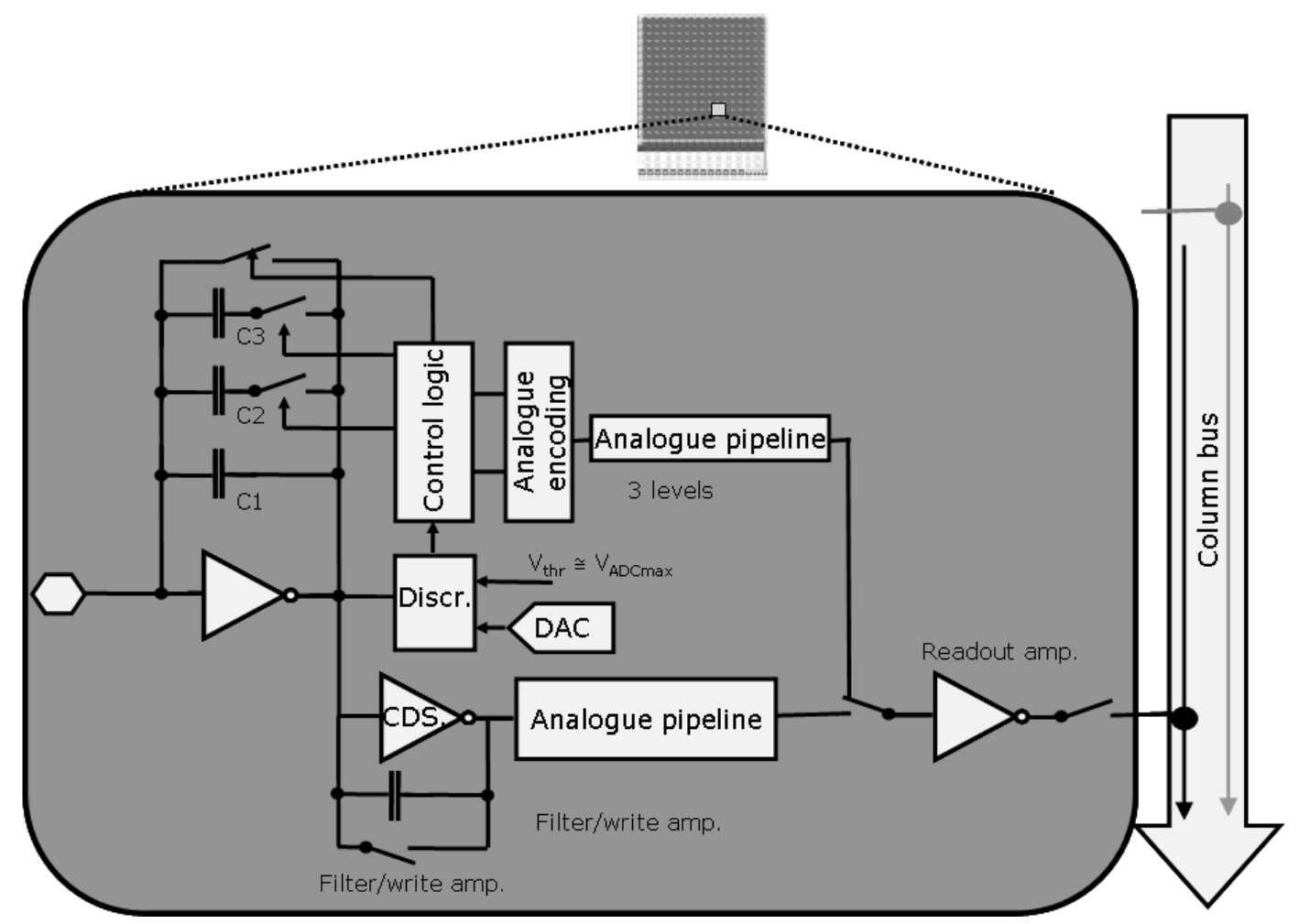

138 Fig.1: pixel stucture of the AGIPD readout ASIC 



139 Fig.2: memory cell stucture embedded in the pixel 


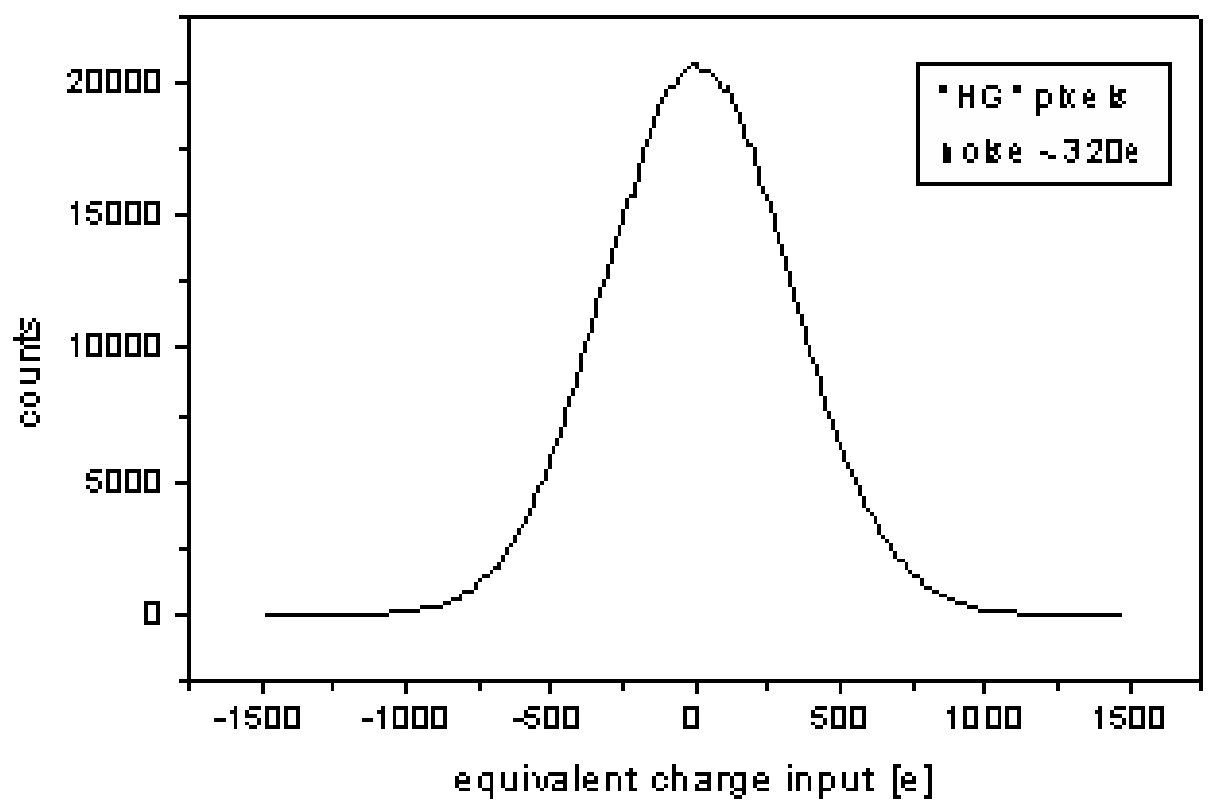

140 Fig.3: noise measurement on AGIPD04 prototype (room temperature) 


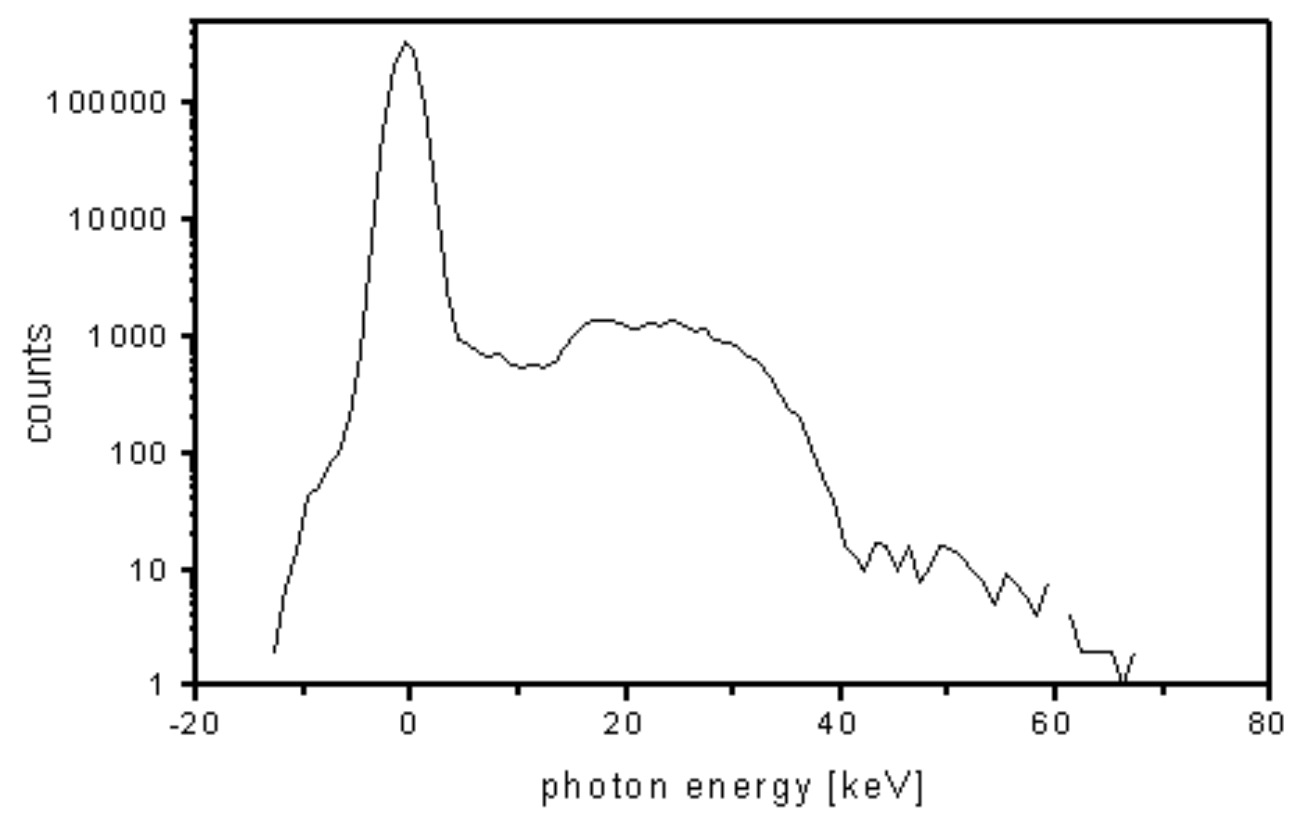

${ }_{141}$ Fig.4: response to Molibdenum target irradiated with X-ray tube 


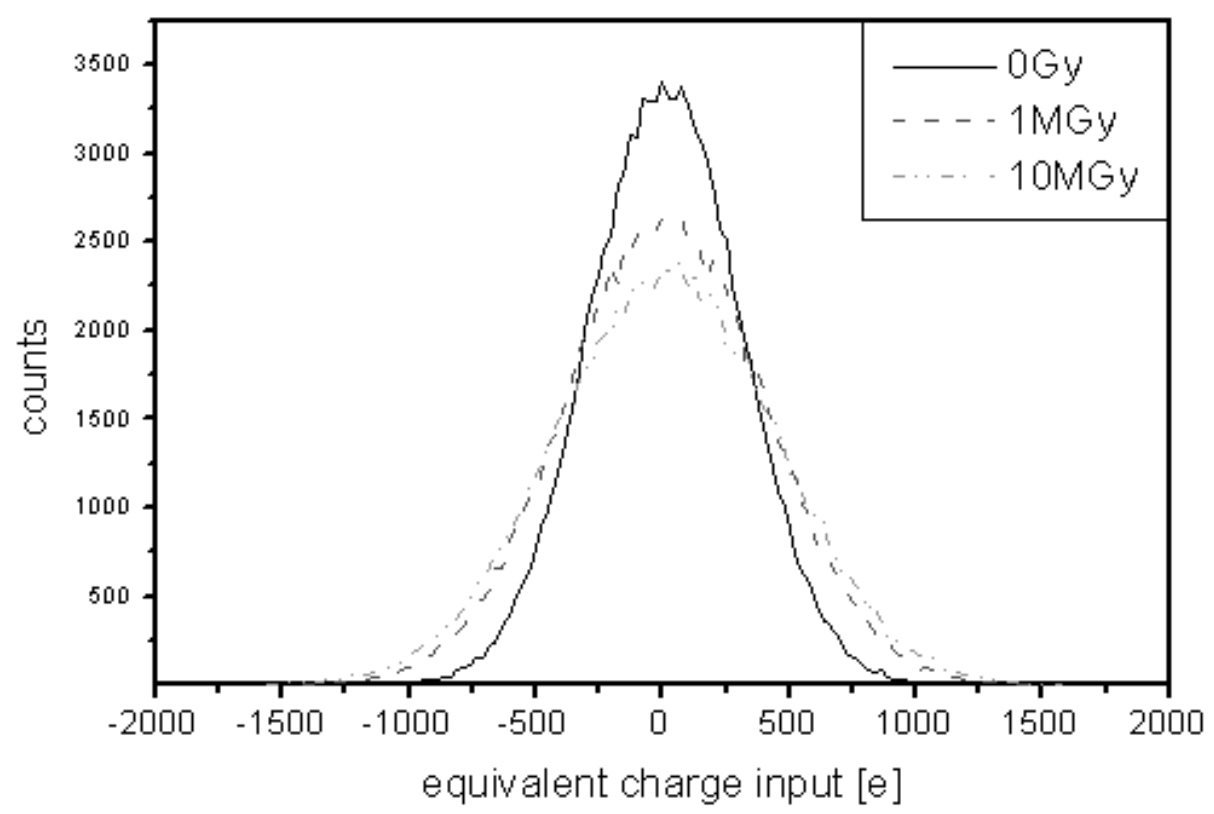

${ }_{142}$ Fig.5: noise measurement on irradiated AGIPD04 prototype (room tempera143 ture) 